\title{
Identification of Saccharomyces cerevisiae strains for alcoholic fermentation by discriminant factorial analysis on electronic nose signals
}

\author{
Montserrat Calderon-Santoyo \\ Laboratorio de Investigación Integral en Alimentos \\ Instituto Tecnológico de Tepic \\ Tepic, Nayarit. Mexico \\ Pascale Chalier \\ UMR 1208 Ingénierie des Agropolymères et des Technologies Emergentes \\ Université Montpellier 2 \\ Place E. Bataillon, Montpellier, France Cedex 5 \\ Dominique Chevalier-Lucia \\ UMR 1208 Ingénierie des Agropolymères et des Technologies Emergentes \\ Université Montpellier 2 \\ Place E. Bataillon, Montpellier, France Cedex 5 \\ Charles Ghommidh \\ UMR Démarche intégrée pour l'obtention d'aliments de qualité \\ Université Montpellier 2 \\ Place E. Bataillon, Montpellier, France Cedex 5 \\ Juan Arturo Ragazzo-Sanchez* \\ Laboratorio de Investigación Integral en Alimentos \\ Instituto Tecnológico de Tepic \\ Tepic, Nayarit. Mexico \\ E-mail: arturoragazzo@hotmail.com
}

Keywords: alcoholic fermentation, discrimination, electronic nose, gas sensor, yeasts.

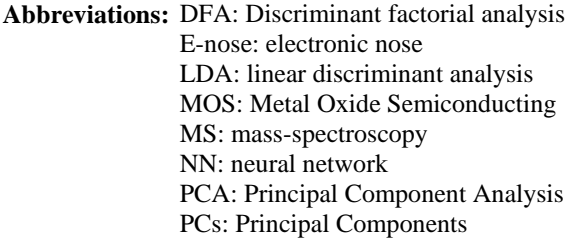

An electronic nose (E-nose) coupled to gas chromatography was tested to monitor alcoholic fermentation by Saccharomyces cerevisiae ICV-K1 and Saccharomyces cerevisiae T306, two strains well-known for their use in oenology. The biomass and ethanol concentrations and conductance changes were measured during cultivations and allowed to observe the standard growth phases for both yeast strains. The two strains were characterized by a very similar tendency in biomass or ethanol production during the fermentation. E-nose was able to establish a kinetic of the production of aroma compounds production and which was then easy to associate with the fermentation phases. Principal Component Analysis (PCA) showed that the data collected by E-nose during the fermentation mainly contained cultivation course information. Discriminant factorial analysis (DFA) was able to clearly identify differences between the two strains using the four main principal components of PCA as input data. Nevertheless, the electronic nose responses being mainly influenced by cultivation course, a specific data treatment limiting the time influence on data was carried out and permitted to achieve an overall performance of $83.5 \%$.

Electronic noses (E-noses) are tested and applied since eighty's as aromatic quality sensors in the agricultural, environmental, medical, biotechnological and food domains (Bourgeois et al. 2001; Ampuero and Bosset, 2003; Thaler and Hanson, 2005; Rudnitskaya and Legin, 2008; Peris and Escuder-Gilabert, 2009). They are typically composed of an array of non-specific chemical gas sensors characterized by a broad and partly overlapping selectivity to volatile compounds. This concept was inspired by the human nose

*Corresponding author 
and clearly shows similarity with the human brain-olfactory system (Gardner and Barlett, 1994). Nevertheless, E-nose has large differences both in sensibility and selectivity compared to the human nose. In fact, researchers tend to consider this technology as an efficient alternative to sensory analysis. Besides, the conventional flavour analysis methods such as mass spectroscopy combined with gas chromatography or high pressure liquid chromatography provide information on aroma analysis and recognition, but are rather time-consuming and expensive. E-nose is on the contrary a fast, reliable, cost-effective, in line, automatic and operator-friendly system of aroma analysis (Peris and Escuder-Gilabert, 2009; Yu et al. 2009b). Nevertheless, the E-nose's gas sensors provide a large and complex amount of data (i.e. sensor responses), which has to be processed by pattern recognition techniques such as principal component analysis (PCA), linear discriminant analysis (LDA) or neural network (NN) (Hernández Gómez et al. 2006; Scott et al. 2006; Ragazzo-Sanchez et al. 2008; Zhang et al. 2008). Recently, several studies proposed to improve discrimination between very similar E-nose data by first of all analyzing E-nose data by PCA, in order to reduce the data dimension, and secondly, selecting some of the more most relevant principal component values as input in classification techniques such as LDA or NN (Yu et al. 2009a; Yu et al. 2009b). Data processing improves the selectivity of the systems leading to an extensive range of applications.

Samples classification (Shaw et al. 2000; Ragazzo-Sanchez et al. 2008; Tudu et al. 2009), adulterations or detection of defaults in aroma (Aparicio et al. 2000; Hai and Wang, 2006; Yu et al. 2007), quality measurement (Sarig, 2000; Yu et al. 2009a) and process monitoring (Bhattacharyya et al. 2007; Gutiérrez-Mendez et al. 2008) are the main applications of the E-nose technology. Recent applications of E-nose concerned the biotechnological domain. E-nose was implemented to study its ability for diagnosis, detection and screening of various stages of renal disease (Haick et al. 2009) or for monitoring industrial processes related to microorganisms (Bachinger et al. 2001; Clemente et al. 2008) or cells cultures (Bachinger et al. 2000; Bachinger et al. 2002; Kreij et al. 2005). In the latter areas, the initial studies consisted in analyzing the headspace generated by various microorganisms grown on Petri dishes by the E-nose and detecting and identifying microorganisms from the responses of the E-nose treated by chemometrics (Dutta et al. 2002; Moens et al. 2006). For instance, Dutta et al. (2002) showed that gas sensors efficiently identified six species of bacteria responsible for eye infections and ten clinically important microorganisms were successfully tested and identified by Moens et al. (2006). Different studies were carried out during microorganisms cultivations and not after a definite incubation time. Gardner et al. (1998) successfully predicted the class and growth phase of two potentially pathogenic bacteria by analyzing samples of the cultivation headspace with six Metal Oxide Semiconducting (MOS) gas sensors. Classification was performed with a multi- layer perceptron network applied on the responses of the Enose pre-treated by different pre-processing and normalization methods. A cultivation of Saccharomyces cerevisiae on glucose was monitored on-line (ethanol concentration and course cultivation) analyzing the cultivation gas effluent with the E- nose (Liden et al. 2000). The potential of the E-nose technology was confirmed as well on a production-scale CHO-cell process (Bachinger et al. 2000), on the detection of the metabolic burden on a recombinant E. coli strain (Bachinger et al. 2001) or bacterial infections in cell cultures (Bachinger et al. 2002; Kreij et al. 2005). Brandgård et al. (2001) successfully monitored growth of Methanobacterium formicicum using a MOS and MOSFET (Metal Oxide Semiconducting Field Effect Transistor) E-nose in order to detect disturbances in the microbiological process. The gas sensor array technology was applied on complex fermentation medium such as milk (Magan et al. 2001), to investigate yogurt (Cimander et al. 2002; Navratil et al. 2004), wine (Pinheiro et al. 2002) or black tea (Bhattacharyya et al. 2007) fermentations. In the case of wine, Pinheiro et al. (2002) analyzed wine-must fermentation with an E-nose insisting on sample pre-treatment (organophilic pervaporation) to really detect changes in aroma compound and not only in ethanol concentration during fermentation. In fact, in the case of alcoholic fermentations or alcoholic beverages analysis, the high ethanol concentration tends to affect the detection of aroma compounds by the E-nose technology (Ragazzo-Sanchez et al. 2004; Lozano et al. 2007; Ragazzo-Sanchez et al. 2008; Peris and Escuder-Gilabert, 2009). However, various de-alcoholisation techniques have been developed, such as purge and trap, dynamic headspace, distillation, adsorption methods (SPME), membrane systems, liquid-liquid extraction and massspectroscopy (MS)-based E-nose (Peris and EscuderGilabert, 2009). Marti et al. (2004) used an E-nose system associated to a headspace sampler coupled to a mass spectrometer to successfully discriminate wines according to different oenological parameters. In this system, ethanol interferences were avoided due to selection of the proper

Table 1. Name and arrangement of sensors in the three temperature-controlled chambers of the E-nose.

\begin{tabular}{|c|c|c|}
\hline Chamber 1 & Chamber 2 & Chamber 3 \\
\hline T30/1 & $\mathrm{P} 30 / 1$ & SY/LG \\
\hline $\mathrm{P} 10 / 1$ & $\mathrm{P} 40 / 2$ & $\mathrm{SY} / \mathrm{G}$ \\
\hline $\mathrm{P} 10 / 2$ & $\mathrm{P} 30 / 2$ & $\mathrm{SY} / \mathrm{AA}$ \\
\hline $\mathrm{P} 40 / 1$ & $\mathrm{~T} 40 / 2$ & $\mathrm{SY} / \mathrm{Gh}$ \\
\hline $\mathrm{T} 70 / 2$ & $\mathrm{~T} 40 / 1$ & $\mathrm{SY} / \mathrm{gCTI}$ \\
\hline $\mathrm{PA} 2$ & $\mathrm{TA2}$ & $\mathrm{SY} / \mathrm{gCT}$ \\
\hline
\end{tabular}


fragment-ion range for the MS. Another solution was proposed by Ragazzo-Sanchez et al. (2004) who developed a back-flush gas chromatography to pre-treat vapour samples and totally and rapidly remove alcohol from samples before the analysis with the E-nose. In this case, the E-nose system associated to PCA allowed to discriminate four alcoholic beverages (red wine, tequila, vodka and whisky) and detect four compounds responsible for off-flavour in red wine (Ragazzo-Sanchez et al. 2005; Ragazzo-Sanchez et al. 2008; Ragazzo-Sanchez et al. 2009).

The aim of this study was to investigate on-line alcoholic fermentations with an E-nose equipped with a back-flush gas chromatography removing alcohol from samples before analyzing. A second objective was to discriminate two different oenological Saccharomyces cerevisiae strains using the E-nose responses whatever the cultivation time was.

\section{MATERIALS AND METHODS}

\section{Microbial strains and medium}

Two oenological Saccharomyces cerevisiae strains (ICVK1 and T306), supplied by l'Institut Coopératif du Vin (ICV-Montpellier), were obtained as active dry yeasts and used for culture experiments. A synthetic medium previously described by Bely et al. (1990) was used for fermentations. The strains were inoculated in bioreactor in a proportion of $50 \mathrm{mg}$ dry matter/L. One gram of dry yeast was rehydrated in a sterile flask in $10 \mathrm{~mL}$ of stirred distilled water at $32^{\circ} \mathrm{C}$ for $15 \mathrm{~min}$. Then, $0.75 \mathrm{~mL}$ of this preinoculum was added to $200 \mathrm{~mL}$ of the synthetic medium to carry out the final inoculum.

\section{Fermentation process}

A 2 L bioreactor (Inceltech, Toulouse, France), with a $1.5 \mathrm{~L}$ working volume and equipped with standard measurement and control units for temperature, $\mathrm{pH}$ and stirrer speed, was used to carried out batch alcoholic fermentations. Cultivation stirring and temperature were maintained constant at $200 \mathrm{rpm}$ and $28^{\circ} \mathrm{C}$, respectively. Three different fermentations were carried out for each yeast strain.

\section{Offline analysis}

Biomass. The yeast concentration was determined by filtering $10 \mathrm{~mL}$ of broth through a $0.2 \mu \mathrm{m}$ cellulose nitrate filter (Sartorius, Germany), previously dried and weighed. The filter was then washed twice with distilled water and dried at $102^{\circ} \mathrm{C}$ for $24 \mathrm{hrs}$. The dry cell mass (g of dry cell weight per L) was calculated by weight difference. A fermentation sample was removed from the bioreactor every $10 \mathrm{hrs}$. All samples were measured by triplicate.

Glucose. Glucose was analyzed using a high performance liquid chromatograph (HPLC) (Shimatzu, Japan) on a Aminex HPX87H column (Bio-Rad, CA, USA) at $65^{\circ} \mathrm{C}$. The mobile phase was a $6 \mathrm{mM} \mathrm{H}_{2} \mathrm{SO}_{4}$ solution. Detection was performed with a differential refractive index detector (2410, Waters, Milford, MA, USA).

\section{Online analysis}

Electronic nose. A commercially available E-nose (FOX 4000, AlphaMOS, France) with eighteen different metal oxide semiconductor gas sensors (MOS) was used. The different sensors were disposed in three temperaturecontrolled chambers, each chamber including six sensors, a thermometer and a humidity sensor. The sensor arrangement in each chamber is depicted on Table 1. A generator of purified air (Whatman, UK) with a $\mathrm{CaCl}_{2}$ post dehydration column was used to provide clean dry air to the nose system.

The bioreactor headspace was continuously pumped thanks to a membrane compressor (Fisher Bioblock Scientific, France) placed before the sampling loop. Due to the small bioreactor volume, the gas sample was reintroduced in the bioreactor in order to avoid depression and volatile compounds losses. Sampling from this gas flow was performed every $30 \mathrm{~min}$ through a 6-port automated sampling valve and the sample was introduced in a gas chromatograph (IGC 121C, Intersmat, Belgium) equipped with a Porapak Q column $(1 \mathrm{~m} \times 0.32 \mathrm{~cm})$. The samples were then dehydrated and de-alcoholised by a patented back-flush technique (Ragazzo-Sanchez et al. 2004). In this technique three multiway electro-valves were used for automatic injection in the GC, column back-flush and automatic injection in the E-nose.

Table 2. The confusion matrix showing the strain classification by DFA using the front four principal components obtained from the PCA of the corrected data. The accuracy of the classification is defined as $(210+222) /(290+299)$, namely $73.2 \%$.

\begin{tabular}{|l|l|l|l|}
\hline & \multicolumn{1}{|c|}{ True } & \multicolumn{1}{|c|}{ Class } & \\
\hline Predicted class & ICV-K1 (287) & T306 (303) & \% correct \\
\hline ICV-K1 & 210 & 80 & 72.4 \\
\hline T306 & 77 & 222 & 74.2 \\
\hline
\end{tabular}


Ethanol. Ethanol was analyzed on-line thanks to the dehydration-desalcoholisation system by gas chromatography (IGC 121C, Intersmat, Belgium) with a flame ionization detector. The analytical column was a $1 \mathrm{~m}$ Porapak Q column operated at $180^{\circ} \mathrm{C}$. Nitrogen served as carrier gas at a flow rate of $18 \mathrm{~mL} / \mathrm{min}$. The ethanol calibration was carried out using standard ethanol solutions placed in the bioreactor and analyzed in the gas chromatography- E-nose system in the operating culture conditions. This calibration was carried out before each fermentation batch.

Biomass. The determination of cell mass concentration was performed by an optical sensor (653/BT65 model, Wedgewood Technology Inc, CA, USA) measuring medium turbidity. Previously, a calibration curve was carried out in order to transform optic density into biomass concentration (g dry matter/L).

Conductance. At the same time, the conductance determination was ensured by a dual-frequency impedance monitoring device. The system was obtained from Fogale Nanotech (Nîmes, France).

\section{Data analysis}

The software provided with the E-nose system was used to acquire and store the gas sensor array signals. From each sensor signal, the fractional difference was calculated as shown in Equation 1:

$S_{f t}=\frac{S_{\mathrm{max}}-S_{\text {bareline }}}{S_{\text {baseline }}}$

[Equation 1]

where $S_{f d}$ corresponds to the modified signal, $S_{\max }$ to the maximum sensor signal value, and $S_{\text {baseline }}$ to the base line sensor signal value.

Each sensor signal was auto-scaled (i.e. mean-centered and divided by its standard deviation for rescaling with unit variance) to obtain $S_{\mathrm{fdN}}$. The maximum value of $\mathrm{S}_{\mathrm{fdN}}$ for each sensor was used for PCA or DFA to avoid domination of high sensor responses in data processing. Relevant information contained in low sensor responses were thus taken into account in multivariate analysis processing. PCA and DFA were carried out with the chemometrics toolboxes of the software Matlab 6.5 software (the MathWorks Inc, MA, USA).

\section{RESULTS AND DISCUSSION}

\section{Batch cultivations}

Saccharomyces cerevisiae ICV-K1 and Saccharomyces cerevisiae T306 are quite similar in their oenological fermentation characteristics according to the producer (Lallemand, France). They both produce low volatile acidity and sulfate concentration, they are resistant to high alcohol content up to $14 \%$ and $18 \%$ for T306 and ICV-K1, respectively. Nevertheless, they are used for different applications: ICV-K1 strain is recommended as a starter yeast strain for red or white wines while T306 is particularly intended to white aromatic wines. Besides, the ICV-K1 strain has a neutral sensory effect whereas the T306 is inclined to enhance varietal character of grapes. The fermentation performances of the ICV-K1 strain are reported in Figure 1, which shows the biomass, glucose and conductance changes in function of cultivation course. Similar changes were observed for the two other fermentations using the ICV-K1 strain and the three ones with the T306 strain. Based on the biomass concentration changes, the standard growth phases were observed. In particular, three stages are distinguished: a lag phase (Figure 1, phase I), then a swift growth phase (Figure 1, phase II) and a long and progressive deceleration phase (Figure 1, phase III). During the phase II, the broth conductance decreased due to the ammonium nitrogen and mineral ions consumption. During the third phase, an increase in the conductance clearly appeared due to the release of ions and secondary metabolism compounds (such as aroma compounds) by yeasts. The two strains did not clearly have a distinct behaviour in terms of biomass or ethanol concentration during the cultivation as shown in Figure 2.

For all of the fermentation runs and, whatever the strain was, the ethanol concentration had a similar evolution (Figure 2). The same observation was carried out for biomass concentration changes (data no shown). Particularly, neither of these two strains appeared to produce more ethanol or biomass at the end of cultivation. It clearly appeared that the conventional changes of biomass or ethanol during the fermentation did not enable to discriminate the two strains.

\section{Analysis of the electronic nose sensor responses}

The ethanol concentration was high (from 0 g.L $\mathrm{L}^{-1}$ to 90 g.L $\mathrm{L}^{-}$ ${ }^{1}$ ) at the end of these oenological fermentations as shown in Figure 2. This phenomenon induced the use of a dehydration and de-alcoholisation system to avoid saturating sensors and masking the minor volatile compounds. The back-flush system proposed by Ragazzo et al. (2004) enabled to remove the interference due to ethanol without eliminate aroma compounds of interest. This system has the advantage of, first of all, separating the different compounds in the GC column, and then, by reverting to the gas vector inside the column after water and ethanol elution, only collecting and introducing the other volatile compounds in the E-nose system. Figure 3 presents the typical time profiles of selected sensor signals from the E-nose for the ICV-K1 strain. Only two successive phases appeared in these sensor profiles in comparison with the three phases observed during the yeast cultivation. The sensor responses remained constant until 22 hrs of cultivation. This was associated with the lag phase and the fast growth phase: these two cultivation steps were 
Table 3. The confusion matrix showing the strain classification using the front four principal components of PCA of the normalized data. The accuracy of the classification is defined as $(252+240) /(290+299)$, namely $83.5 \%$.

\begin{tabular}{|l|l|l|l|}
\hline & \multicolumn{1}{|c|}{ True } & \multicolumn{1}{|c|}{ Class } & \\
\hline Predicted class & ICV-K1 (287) & T306 (303) & \% correct \\
\hline ICV-K1 & 252 & 38 & 86.9 \\
\hline T306 & 59 & 240 & 80.3 \\
\hline
\end{tabular}

characterized by a very low release of aroma compounds. The sensor responses then clearly increased as soon as the long deceleration phase began it is associated with a large aroma compounds production.

\section{Principal component analysis}

The aim of Principal Component Analysis (PCA) is to transform the numerous original variables (sensor signals) into new variables, Principal Components (PCs), which are linear combinations of the original ones. In fact, the inherent structure of data set is preserved while its resulting variance is maximized. In a first step, the E-nose responses for each strain were analyzed separately by PCA. Figure 4 shows the score plot of the two main principal components for a T306 fermentation. The accumulated contribution of the two PCs was $75.4 \%$. The first component (PC1) accounted for $62.9 \%$ of the total variance, it was then the predominating direction among the others components. Figure 4 clearly showed that the visualized information essentially concerned the course of fermentation and strongly suggested that the direction of component PC1 would demonstrate the time scale of the culture. The score plot defined by the front two principal components showed three different groups: the first one (A on Figure 4) corresponded to the measurements carried out during the lag and fast growth phases (from 0 to $22 \mathrm{hrs}$ ), the second zone (B on Figure 4, from 22 to 33 hrs) was attributed to the main aroma compounds release stage and the third one (Figure 4C, 34 hrs to the fermentation end) was related to the progressive deceleration phase. Whatever the yeast strain was, similar observations were made upon PCA for the other fermentation runs.

The loading plot for the same fermentation is presented on Figure 5. A strong positive correlation between chamber 1 and 2 (except one "T40/1") sensors and PC 1 indicated that these sensors were certainly the more sensitive to aroma compounds released by the two yeast strains used in the study.

In a second stage, PCA was carried out on the E-nose data about fermentations from the two different yeast strains together. Whatever the fermentation run chosen, similar results were observed. A bi-dimensional plot of the samples scores (third fermentation with ICV-K1 and second fermentation with T306) in the space defined by the two main principal components showed a distinct location of ICV-K1 and T306 fermentation samples (Figure 6). Besides, in each strain groups, three sub-groups, as described above, function of the time course of cultivation were observed. Nevertheless, the location of T306 samples appeared more compact than the location of ICV-K1 samples. The second principal component was probably less discriminant for the T306 strain during the cultivation course. Nevertheless, the ICV-K1 strain has the property to having a neutral sensory effect and certainly produces lower amounts of aroma compounds than to the T306 strain in the same cultivation conditions.

The PCA showed that the collected E-nose data mainly contained chronological information. The two strains were distinguishable on score plots of PCA and it would be interesting to investigate if the E-nose could be a predictive tool and not only a descriptive one.

\section{Classification of yeast stains}

The main objective was to predict the yeast strain involved in a fermentation process analyzing a sample removed from the reactor at any time and introduce in the E-nose system. As it seemed obvious to use non-redundant variables in discriminant factorial analysis (DFA), the four main components of PCA were extracted and used as uncorrelated input variables in DFA. This methodology enabled to decrease the input data dimension and optimize the feature vector (Yu et al. 2009a). These front four principal components extracted by PCA contributed to $93,6 \%$ of total variance of all the samples.

The overall performance of DFA with the front four PC values was appreciated through a confusion matrix (Table 2) where the diagonal indicated the correctly identified samples (in bold). The correct classification ratio was $73.2 \%$. This moderate result indicated that an improvement of the treatment of the E-nose data before the DFA was necessary.

Particularly, as the time course of fermentation was the most important information in the PC1, that did not enable to obtain a better classification. A further step of the study was thus to modify the E-nose data in order to eliminate the 
time influence before carrying out DFA. The correlation coefficient between each sensor and the biomass and ethanol concentration was studied. The three most correlated sensors with these two cultivation parameters, strongly time dependent, were "P40/1", "P10/1" and "P40/2". The sensor responses were then normalized by dividing each response of each sensor by the sum of the three former sensors responses at the same experimental time in order to remove the time influence in sensor responses. Figure 7 and Figure 8 present the score plot and loading plot of the PCA carried out with the normalized data corresponding to a fermentation of each strain. As shown in Figure 7, a better description was obtained than previously and the two strains were clearly separated along the abscise axis. "T30/1" and "T70/2" seemed to be particularly correlated to ICV-K1 released components, as indicated by the loading plot (Figure 8). This normalization step enabled to have chronological information on PC1 axis and to collect information related to strain on PC2. The time influence on data was then attenuated after the normalization step. LDA was performed with the front four PCs as previously described for non-normalized data. The confusion matrix presented in Table 3 was obtained after DFA by cross validation and the correct classification achieved $83.5 \%$. The classification was particularly improved for the ICV-K1 strain (correct classification ratio: 86.9\%) even though this strain had a rather neutral sensory effect during wine fermentation.

\section{CONCLUDING REMARKS}

This work showed that E-nose is both a descriptive and predictive tool for the fermentation monitoring, even for alcoholic fermentation thanks to a dehydration and dealcoholisation system coupling a GC and E-nose. PCA of E-nose responses appeared to be mainly influenced by cultivation course. LDA using the front four PC values of the PCA as input data had not successfully discriminat the two strains ICV-K1 and T306. A specific data treatment of normalization to reduce the time's influence on the E-nose data was proposed to obtain information related more particularly to yeast strain differences. When the front four principal components of PCA with normalized data were used as input in LDA, the strain classification was improved although the two strains have very similar fermentation characteristics.

\section{REFERENCES}

AMPUERO, S. and BOSSET, J.O. The electronic nose applied to dairy products: a review. Sensors and Actuators B: Chemical, August 2003, vol. 94, no. 1, p. 1-12.

APARICIO, Ramón; ROCHA, Silvia M.; DELGADILLO, Ivonne and MORALES, María Teresa. Detection of rancid defect in virgin olive oil by the electronic nose. Journal of Agricultural and Food Chemistry, March 2000, vol. 48, no. 3, p. 853-860.
BACHINGER, T.; MANDENIUS, C.F.; STRIEDNER, G.; CLEMENTSCHITSCH, F.; DÜRRSCHMID, E.; CSERJAN-PUSCHMANN, M.; DOBLHOFF-DIER, O. and BAYER, K. Non-invasive detection of the metabolic burden on recombinant microorganisms during fermentation processes. Journal of Chemical Technology and Biotechnology, August 2001, vol. 76, no. 8, p. 885-889.

BACHINGER, Thomas; RIESE, Ulrich; ERIKSSON, Rolf and MANDENIUS, Carl-Fredrik. Monitoring cellular state transitions in a production-scale CHO-cell process using an electronic nose. Journal of Biotechnology, January 2000, vol. 76, no. 1, p. 61-71.

BACHINGER, Thomas; RIESE, Ulrich; ERIKSSON, Rolf and MANDENIUS, Carl-Fredrik. Gas sensor arrays for early detection of infection in mammalian cell culture. Biosensors and Bioelectronics, May 2002, vol. 17, no. 5, p. 395-403.

BELY, Marina; SABLAYROLLES, Jean-Marie and BARRE, Pierre. Automatic detection of assimilable nitrogen deficiencies during alcoholic fermentation in enological condition. Journal of Fermentation and Bioengineering, 1990, vol. 70, no. 4, p. 246-252.

BHATTACHARYYA, Nabarun; SETH, Sohan; TUDU, Bipan; TAMULY, Pradip; JANA, Arun; GHOSH, Devdulal; BANDYOPADHYAY, Rajib; BHUYAN, Manabendra and SABHAPANDIT, Santanu. Detection of optimum fermentation time for black tea manufacturing using electronic nose. Sensors and Actuators B: Chemical, March 2007, vol. 122, no. 2, p. 627-634.

BOURGEOIS, Wilfrid; BURGESS, Joanna E. and STUETZ, Richard M. On-line monitoring of wastewater quality: a review. Journal of Chemical Technology and Biotechnology, April 2001, vol. 76, no. 4, p. 337-348.

BRANDGÅRD, Jennie; SUNDH, Ingvar; NORDBERG, Åke; SCHNÜRER, Anna; MANDENIUS, Carl-Fredrik and MATHISEN, Berit. Monitoring growth of the methanogenic archaea Methanobacterium formicicum using an electronic nose. Biotechnology Letters, February 2001, vol. 23, no. 4, p. 241-248.

CIMANDER, Christian; CARLSSON, Maria and MANDENIUS, Carl-Fredrik. Sensor fusion for on-line monitoring of yoghurt fermentation. Journal of Biotechnology, November 2002, vol. 99, no. 3, p. 237-248.

CLEMENTE, J.J.; MONTEIRO, S.M.S.; CARRONDO, M.J.T. and CUNHA, A.E. Predicting sporulation events in a bioreactor using an electronic nose. Biotechnology and Bioengineering, October 2008, vol. 101, no. 3, p. 545-552.

DUTTA, Ritaban; HINES, Evor L.; GARDNER, Julian W. and BOILOT, Pascal. Bacteria classification using Cyranose 320 electronic nose. BioMedical Engineering OnLine, October 2002, vol. 1, no. 4. 
GARDNER, Julian W. and BARLETT, Philip N. A brief history of electronic noses. Sensor and Actuators B: Chemical, March 1994, vol. 18, no. 1-3, p. 210-211.

GARDNER, J.W.; CRAVEN, M.; DOW, C. and HINES, E.L. The prediction of bacteria type and culture growth phase by an electronic nose with a multi-layer perceptron network. Measurement Science and Technology, January 1998, vol. 9, no. 1, p. 120-127.

GUTIÉRREZ-MÉNDEZ, N.; VALLEJO-CORDOBA, B.; GONZÁLEZ-CÓRDOVA, A.F.; NEVÁREZMOORILLÓN, G.V. and RIVERA-CHAVIRA, B. Evaluation of aroma generation of Lactococcus lactis with an electronic nose and sensory analysis. Journal of Dairy Science, January 2008, vol. 91, no. 1, p. 49-57.

HAI, Zheng and WANG, Jun. Electronic nose and data analysis for detection of maize oil adulteration in sesame oil. Sensors and Actuators B: Chemical, December 2006, vol. 119, no. 2, p. 449-455.

HAICK, Hossam; HAKIM, Meggie; PATRASCU, Michael; LEVENBERG, Chen H.; SHEHADA, Nisreen; NAKHOUL, Farid and ABASSI, Zaid. Sniffing chronic renal failure in rat model by an array of random networks of single-walled carbon nanotubes. ACS Nano, May 2009, vol. 3, no. 5, p. 1258-1266.

HERNÁNDEZ GÓMEZ, Antihus; HU, Guixian; WANG, Jun and GARCÍA PEREIRA, Annia. Evaluation of tomato maturity by electronic nose. Computers and Electronics in Agriculture, October 2006, vol. 54, no. 1, p. 44-52.

KREIJ, Karl; MANDENIUS, Carl-Fredrik; CLEMENTE, João J.; CUNHA, Antonio E.; MONTEIRO, Sandra M.S.; CARRONDO, Manuel J.T.; HESSE, Friedemann; MOLINAS, Maria Milagros; WAGNER, Roland; MERTEN, Otto-Wilhelm; GENY-KATINGER, Cécile; LEGER, Wolfgang; WIESINGER-MAYR, Herbert; MÜLLER, Dethard; KATINGER, Hermann; MARTENSSON, Per; BACHINGER, Thomas and MITROVICS, Jan. On-line detection of microbial contaminations in animal cell reactor cultures using an electronic nose device. Cytotechnology, June 2005, vol. 48, no. 1-3, p. 41-58.

LIDEN, Helena; BACHINGER, Thomas; GORTON, Lo and MANDENIUS, Carl-Fredrik. On-line determination of non-volatile or low-concentration metabolites in a yeast cultivation using an electronic nose. Analyst, 2000, vol. 125, no. 6, p. 1123-1128.

LOZANO, J.; SANTOS, J.P.; GUTIERREZ, J. and HORRILLO, M.C. Comparative study of sampling systems combined with gas sensors for wine discrimination. Sensors and Actuators B: Chemical, October 2007, vol. 126, no. 2, p. 616-623.
MAGAN, Naresh; PAVLOU, Alex and CHRYSANTHAKIS, Ioannis. Milk-sense: a volatile sensing system recognises spoilage bacteria and yeasts in milk. Sensors and Actuators B: Chemical, January 2001, vol. 72, p. 28-34.

MARTI, M. Pilar; BUSTO, Olga and GUASCH, Josep. Application of a headspace mass spectroscopy system to the differentiation and classification of wines according to their origin, variety and ageing. Journal of Chromatography, November 2004, vol. 1057, no. 1-2, p. 211-217.

MOENS, M.; SMET, A.; NAUDTS, B.; VERHOEVEN, J.; IEVEN, M.; JORENS, P.; GEISE, H.J. and BLOCKHUYS, F. Fast identification of ten clinically important microorganisms using an electronic nose. Letters in Applied Microbiology, February 2006, vol. 42, no. 2, p. 121-126.

NAVRATIL, Marián; CIMANDER, Christian and MANDENIUS, Carl-Fredrik. On-line multisensor monitoring of Yogurt and filmjolk fermentations on production scale. Journal of Agricultural and Food Chemistry, February 2004, vol. 52, no. 3, p. 415-420.

PERIS, Miguel and ESCUDER-GILABERT, Laura. A $21^{\text {st }}$ century technique for food control: electronic noses. Analytica Chimica Acta, April 2009, vol. 638, no. 1, p. 115.

PINHEIRO, Carmen; RODRIGUES, Carla M.; SCHÄFER, Thomas and CRESPO, João G. Monitoring the aroma production during wine-must fermentation with an electronic nose. Biotechnology and Bioengineering, March 2002, vol. 77, no. 6, p. 632-640.

RAGAZZO-SÁNCHEZ, J.A.; BALDET, P.; GHOMMIDH, C.; CHALIER, P.; DERATANI, A.; BOULET, J.C.; GRENIER, P.; PERSIN, M.; CROUZET, J.; CHANIÉ, E. and PERRAIN, A. Sampler of volatile components, 2004, European Patent number 02291 627.4.

RAGAZZO-SÁNCHEZ, J.A.; CHALIER, P. and GHOMMIDH, C. Coupling gas chromatography and electronic nose for dehydration and desalcoholization of alcoholized beverages. Application to off-flavour detection in wine. Sensors and Actuators B: Chemical, April 2005, vol. 106, no. 1, p. 253-257.

RAGAZZO-SANCHEZ, J.A.; CHALIER, P.; CHEVALIER, D.; CALDERON-SANTOYO, M. and GHOMMIDH, C. Identification of different alcoholic beverages by electronic nose coupled to GC. Sensors and Actuators B: Chemical, August 2008, vol. 134, no. 1, p. 4348.

RAGAZZO-SANCHEZ, J.A.; CHALIER, P.; CHEVALIER-LUCIA, D.; CALDERON-SANTOYO, M. and GHOMMIDH, C. Off-flavours detection in alcoholic beverages by electronic nose coupled to GC. Sensors and 
Actuators B: Chemical, June 2009, vol. 140, no. 1, p. 2934.

RUDNITSKAYA, Alisa and LEGIN, Andrey. Sensor systems, electronic tongues and electronic noses, for monitoring of biotechnological processes. Journal of Industrial Microbiology and Biotechnology, May 2008, vol. 35, no. 5, p. 443-451.

SARIG, Yoav. Potential application of the artificial olfactory sensing for quality evaluation of fresh produce. Journal of Agricultural Engineering Research, November 2000, vol. 77, no. 3, p. 239-258.

SCOTT, Simon M.; JAMES, David and ALI, Zulfiqur. Data analysis for electronic nose systems. Microchimica Acta, December 2006, vol. 156, no. 3-4, p. 183-207.

SHAW, Philip E.; ROUSEFF, Russell L.; GOODNER, Kevin L.; BAZEMORE, Russell; NORDBY, Harold E. and WIDMER, Wilbur. Comparison of the headspace GC and electronic sensor techniques for classification of processed oranges juices. Lebensmittel Wissenschaft und Technologie, August 2000, vol. 33, no. 5, p. 331-334.

THALER, Erica R. and HANSON, C. William. Medical applications of electronic nose technology. Expert Review of Medical Devices, September 2005, vol. 2, no. 5, p. 559566.

TUDU, Bipan; JANA, Arun; METLA, Animesh; GHOSH, Devdulal; BHATTACHARYVA, Nabarun and BANDYOPADHYAY, Rajib. Electronic nose for black tea quality evaluation by an incremental RBF network. Sensors and Actuators B: Chemical, April 2009, vol. 138, p. 90-95.

YU, Huichun C.; WANG, Jun and XU, Yadan. Identification of adulterated milk using electronic nose. Sensors and Materials, 2007, vol. 19, no. 5, p. 275-285.

YU, Huichun; WANG, Jun; XIAO, Hong and LIU, Miao. Quality grade identification of green tea using the eigenvalues of PCA based on the E-nose signals. Sensors and Actuators B: Chemical, July 2009a, vol. 140, no. 2, p. 378-382.

YU, Huichun; WANG, Yongwei and WANG, Jun. Identification of tea storage times by linear discrimination analysis and back-propagation neural network techniques based on the eigenvalues of principal components analysis of E-nose sensor signals. Sensors, June 2009b, vol. 9, no. 10, p. 8073-8082.

ZHANG, Hongmei; WANG, Jun and YE, Sheng. Prediction of soluble solids content, firmness and $\mathrm{pH}$ of pear by signals of electronic nose sensors. Analytica Chimica Acta, January 2008, vol. 606, no. 1, p. 112-118. 


\section{APPENDIX}

\section{FIGURES}

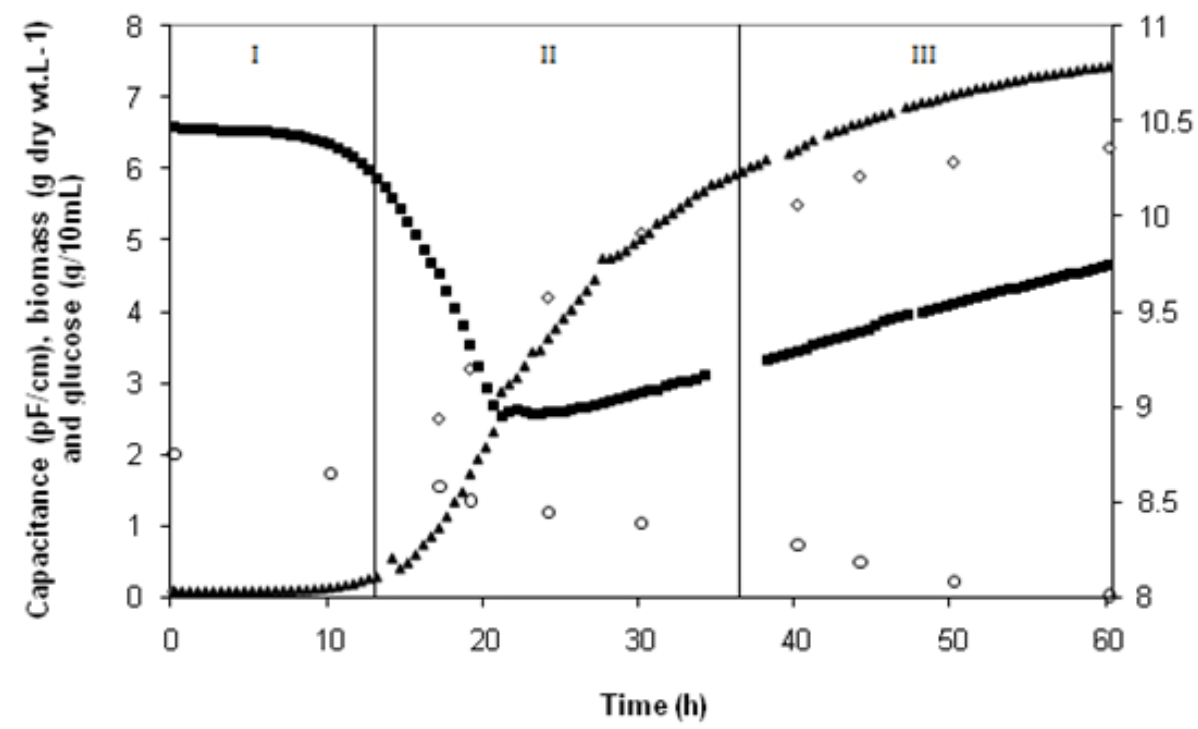

Figure 1. Evolution of the glucose concentration $(O)$, conductance $(\boldsymbol{a})$, on-line (optical) $(\boldsymbol{\Delta})$ and off-line $(>)$ biomass concentrations during fermentation (ICV-K1 strain).

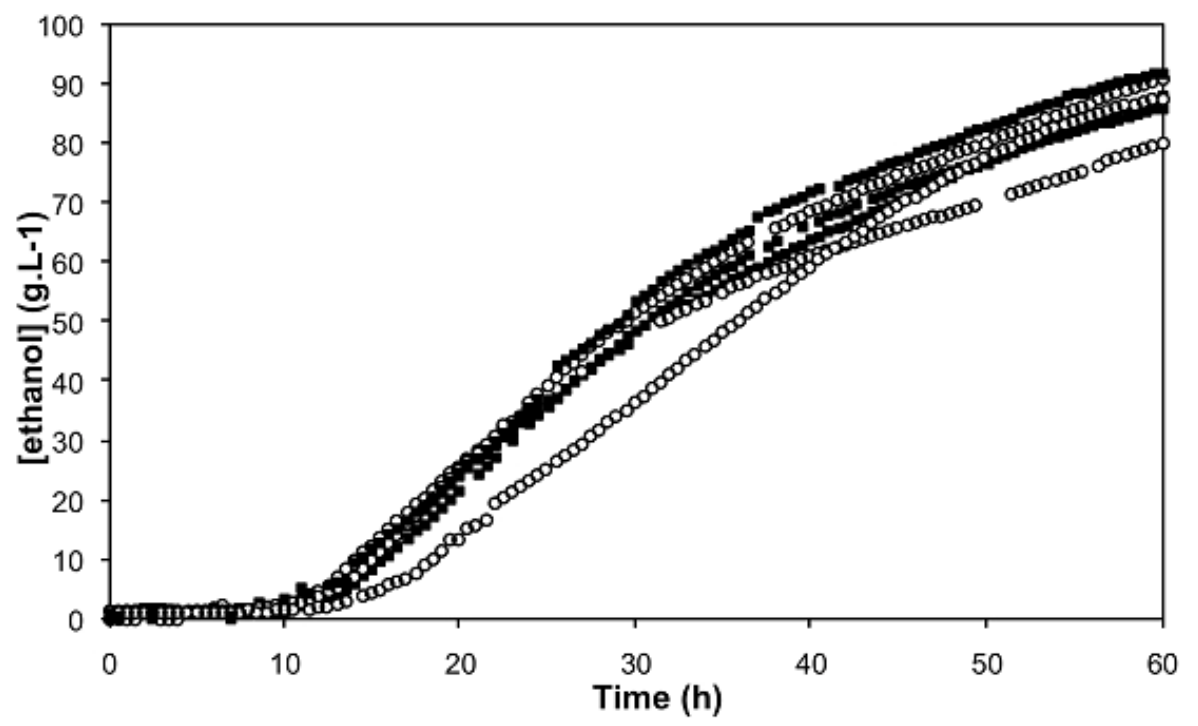

Figure 2. Ethanol concentration $\left(g \cdot \mathrm{L}^{-1}\right)$ changes during the fermentations with $(\square)$ Saccharomyces cerevisiae ICV-K1; and (O) Saccharomyces cerevisiae T-306. 


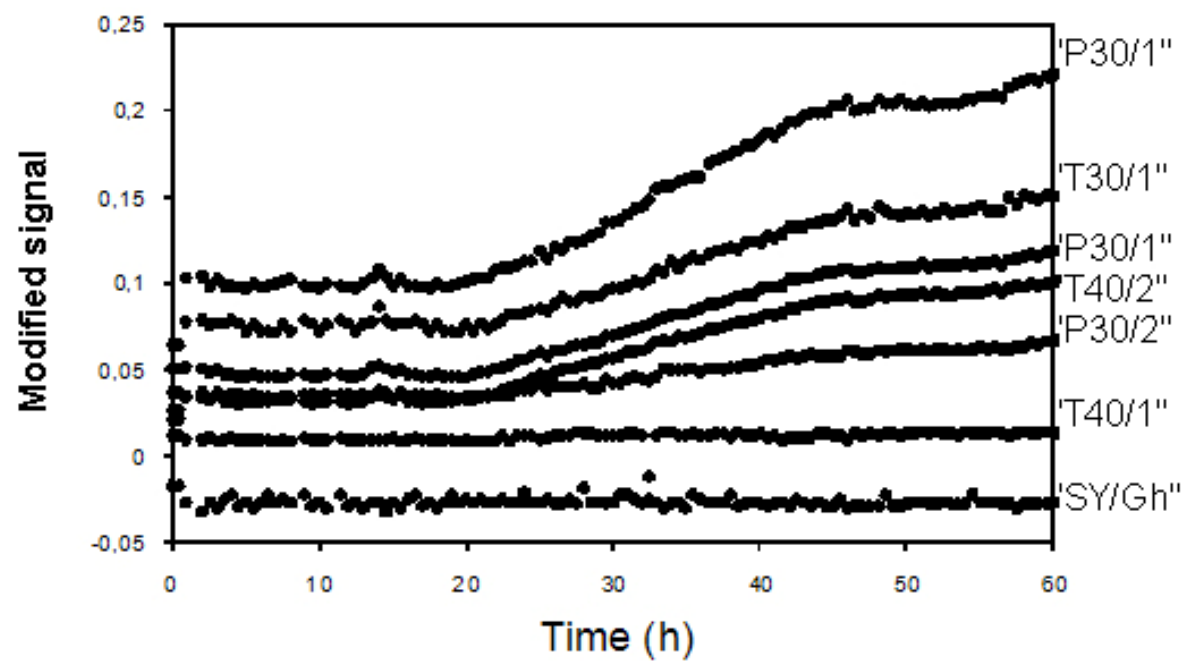

Figure 3. Time profiles of selected MOS sensor signals from the electronic nose for ICV-K1 strain run. Analyze was performed on dehydrated and de-alcoholised headspace of alcoholic fermentation.

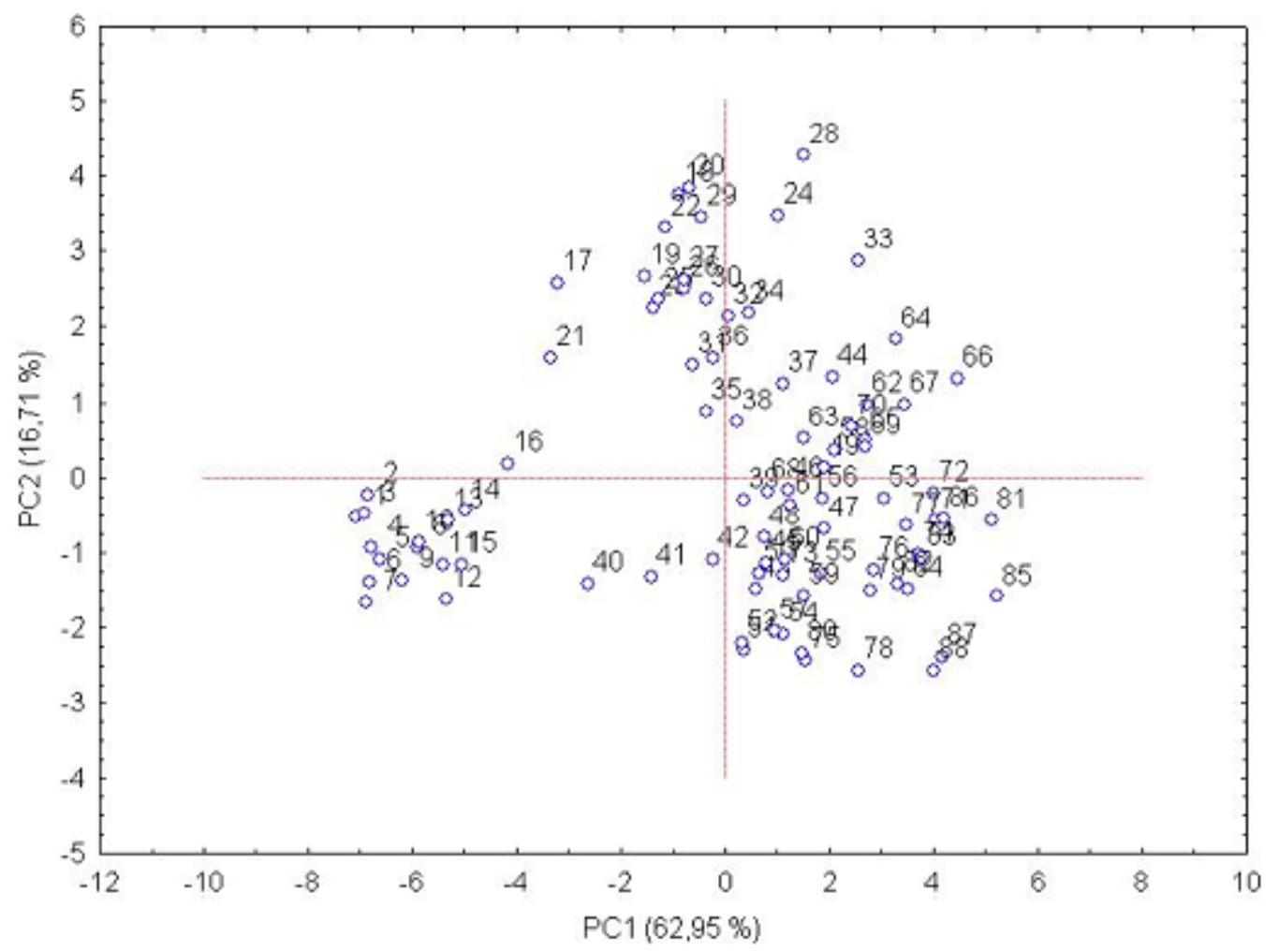

Figure 4. Score plots (PC1 * PC2) of PCA carried out on the E-nose responses of a T306 fermentation. Samples were numbered according to time and one analysis was carried out every $30 \mathrm{~min}$. 


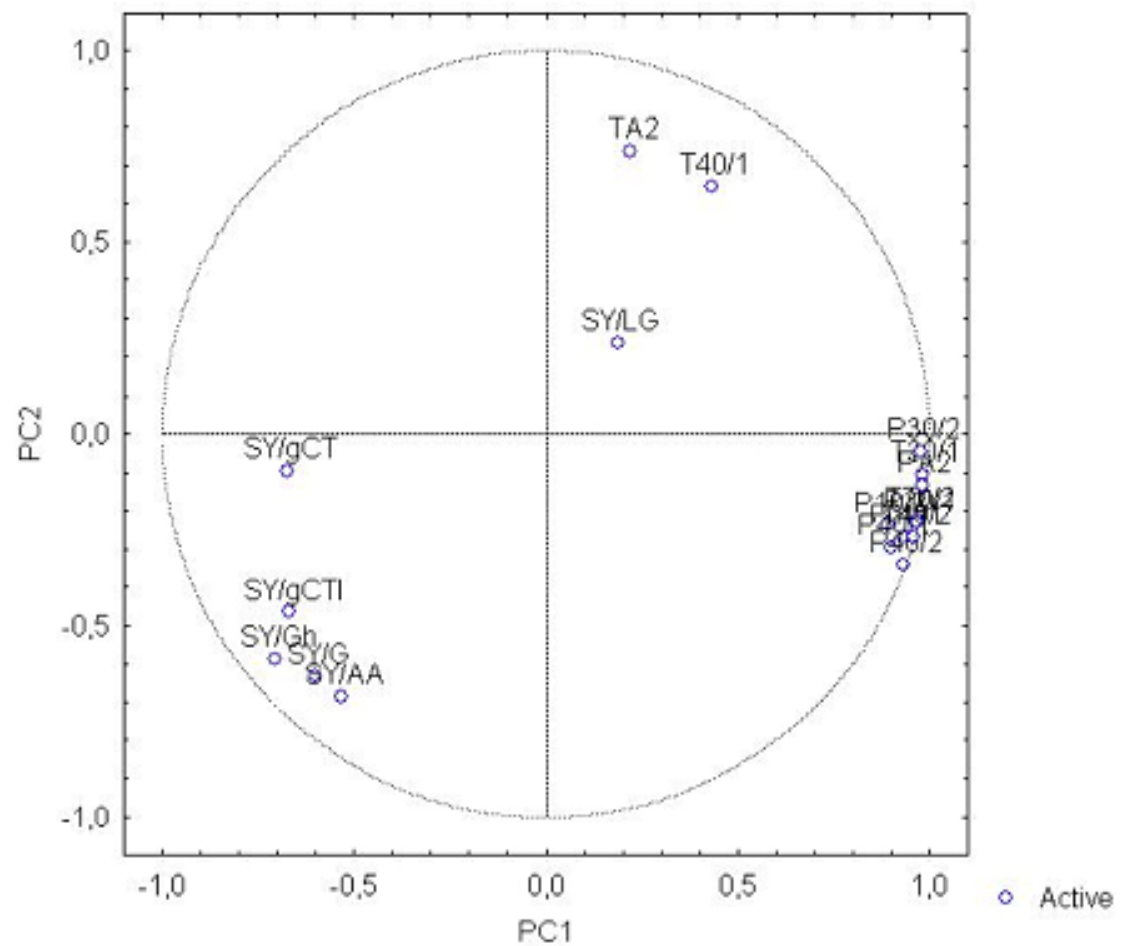

Figure 5. Loading plot of all variables in the plane defined by PC1 * PC2. Data used in PCA corresponded of the E-nose responses from one T306 fermentation.

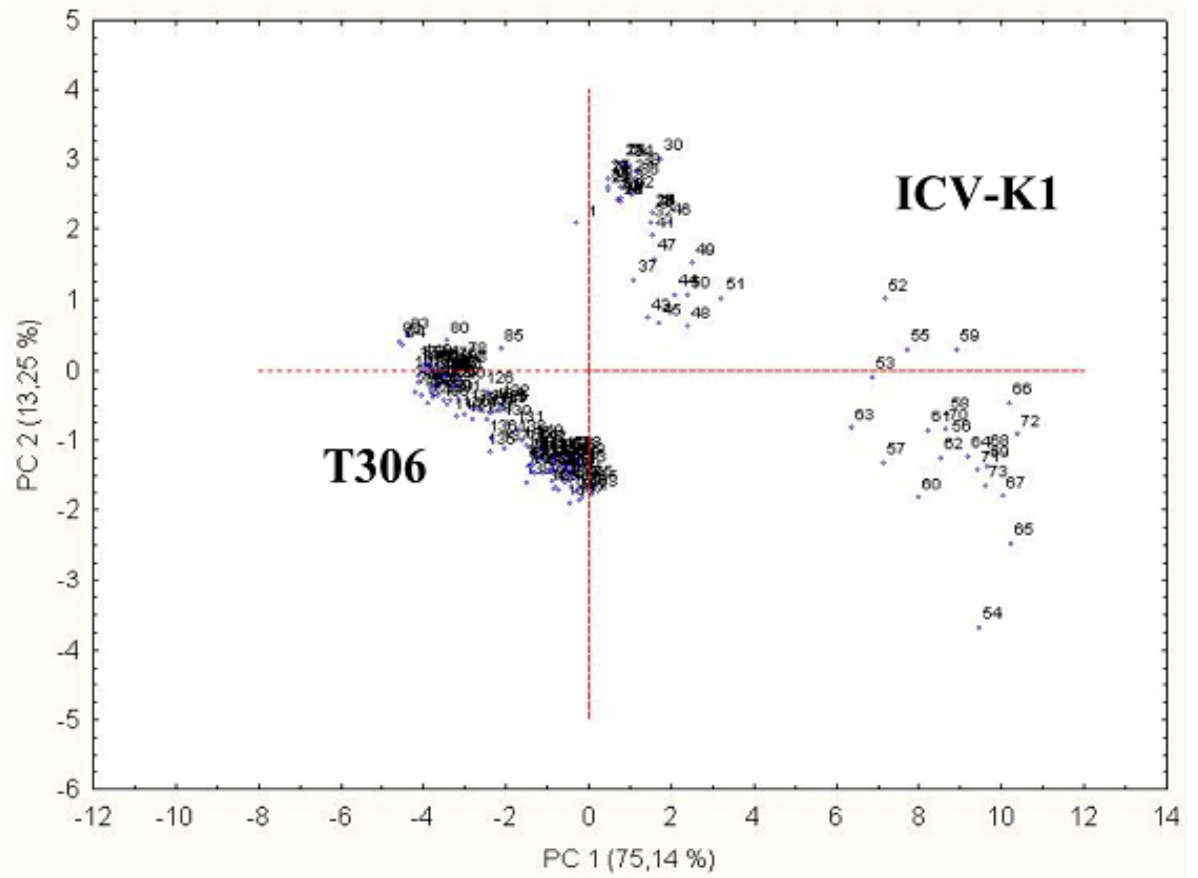

Figure 6. Score plots (PC1 * PC2) of PCA carried out on the E-nose responses for a T306 fermentation and one ICV-K1 fermentation. 


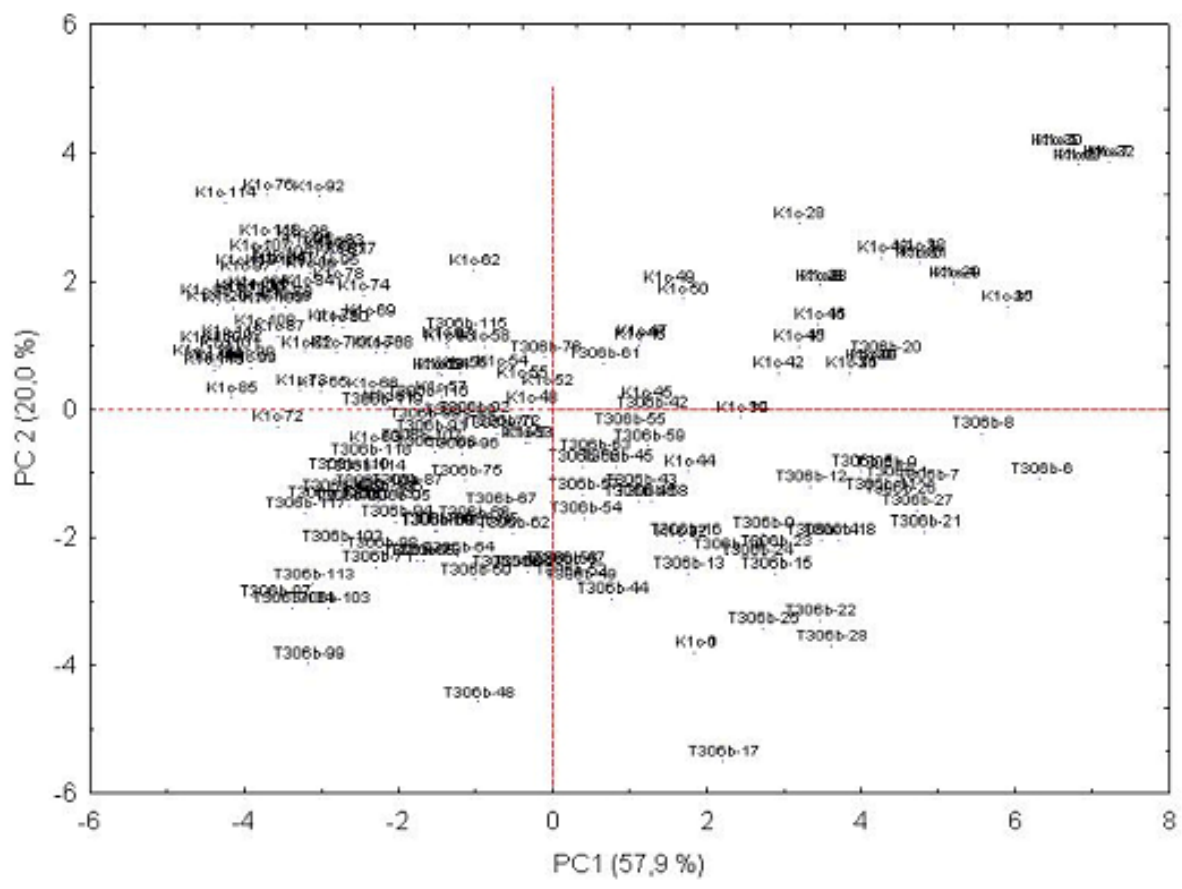

Figure 7. Score plots (PC1 * PC2) of PCA carried out on the E-nose responses for a T306 fermentation and an ICV-K1 fermentation. The E-nose responses were "normalized" as detailed in the text.

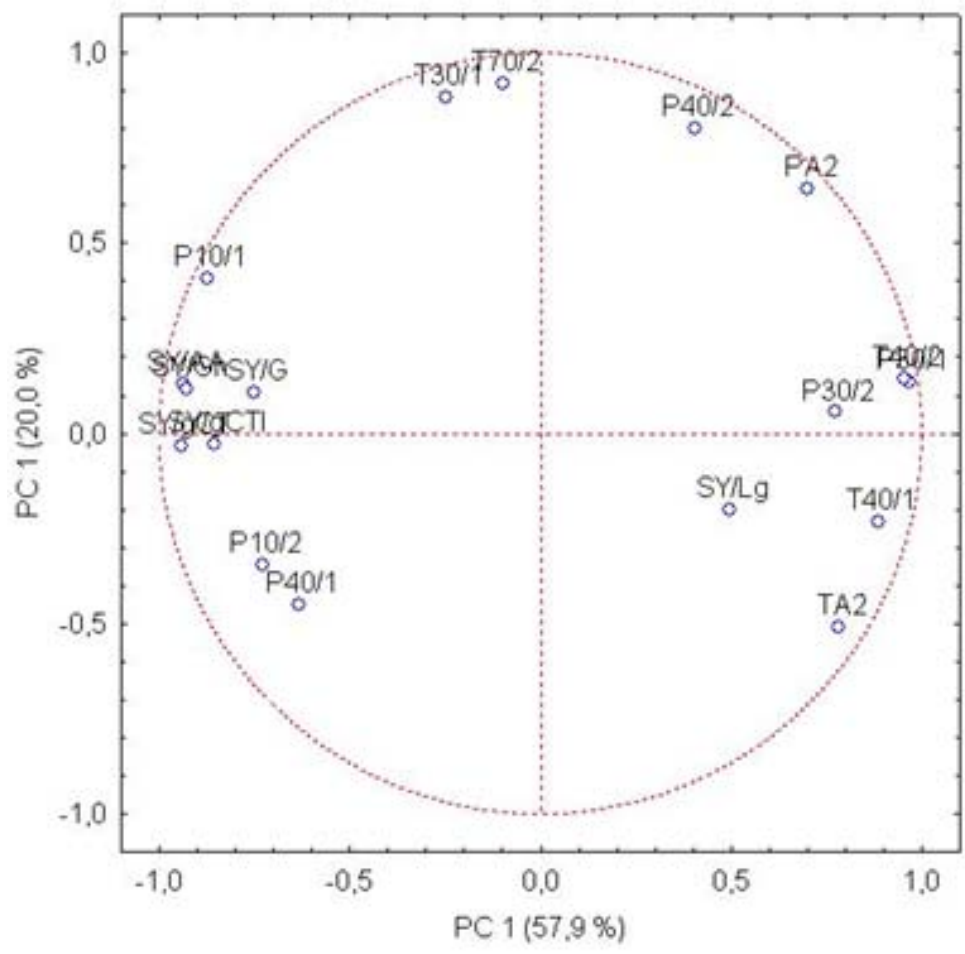

Figure 8. Loading plot of all variables in the plane defined by PC1 * PC2. Data used in PCA corresponded of the E-nose responses for a T306 fermentation and an ICV-K1 fermentation. The E-nose responses were "normalized" as detailed in the text. 